\title{
Tecnura
}

\section{Determinación de los componentes entrópicos de la accidentalidad: el trinomio vehículo/usuario/camino en la metrópoli de Guadalajara, México}

\section{Determination of the entropic components of the accident: the trinomial vehicle- user-road in the metropolis of Guadalajara, Mexico}

Fecha de recepción: 19 de septiembre de 2017

Fecha de aceptación: 28 de noviembre de 2017

\section{Fernando García De Quevedo Najar', Mario Guadalupe Gonzalez Pérez², Yefer Asprilla Lara ${ }^{3}$}

Cómo citar: García de Quevedo N. F., González P., M.G. y Asprilla L., Y. (2018). Determinación de los componentes entrópicos de la accidentalidad: el trinomio vehículo/usuario/camino en la metrópoli de Guadalajara, México. Revista Tecnura, 22(55), 51-65. https://doi.org/10.14483/22487638.13245

\section{Resumen}

Contexto: El reciente incremento de accidentes, percances o siniestros viales en los sistemas urbanos con características metropolitanas ha originado preocupación en las autoridades encargadas de planificar la movilidad y los subsistemas de transporte. Efectivamente, se tiene convergencia que la ocurrencia de este tipo de eventos se aleja de factores puramente estocásticos, que son resultado de anomalías asociadas con la interacción intrasistémica del vehículo, el usuario y el camino. En este sentido, el objetivo de este trabajo consiste en determinar por medio del análisis sistémico los componentes que están provocando entropía en la segunda metrópoli más importante de México.

Método: En este análisis se utilizan los preceptos de la teoría de los sistemas; de tal forma que a través de la triangulación de datos obtenidos in situ y solventados documentalmente se determinan los componentes causales de entropía intrasistémica. Para ello, se elabora una derivación modificada del modelo presión/estado/respuesta que identifica la homeostasia en la que se encuentra el sistema urbano.

Resultados: Las altas tasas de exposición obliga al usuario a ocupar el subsistema vial por largos periodos de tiempo, lo cual detona en errores humanos voluntarios e involuntarios complementados con los requisitos laxos para la obtención de permisos de conducción. De aquí, los componentes que producen accidentes engloban en la parte camino a los pavimentos en mal estado, ausencia de señalización, diseño deficiente de intersecciones, entre otras; en la parte del vehículo, fallas mecánicas por vida útil de los componentes internos, marcos normativos flexibles, etc.; en la parte del usuario por incumplimiento de la normatividad, factores fisiológicos entre otros.

Conclusiones: Desde el análisis sistémico la movilidad es una temática de estudio valiosa para detectar los desequilibrios en el trinomio vehículo/usuario/

1 Ingeniero civil, magister in science in Civil Enginereeng, estudiante de doctorado en Movilidad, Urbana, Transporte y Territorio, Universidad de Guadalajara. Profesor del Instituto Tecnológico de Monterrey. Guadalajara, México. Contacto: fdoquevedo@itesm.mx

2 Ingeniero civil, magíster en Ingeniería Civil, doctor en Ciudad, Territorio y Sustentabilidad. Profesor titular de la Universidad de Guadalajara. Guadalajara, México. Contacto: mario.gonzalez@academico.udg.mx

3 Ingeniero civil, especialista en Infraestructura Vial y Transporte, magíster en Ingeniería Civil, estudiante doctorado en Movilidad, Urbana, Transporte y Territorio, Universidad de Guadalajara. Profesor asociado de la Universidad Distrital Francisco José de Caldas. Bogotá, Colombia. Contacto: yasprillal@udistrital.edu.co 
camino (V-U-C), que nos Ileven a generar medidas preventivas y correctivas que supriman la accidentalidad, la morbilidad y la mortalidad en la metrópolis; o en otros términos para reducir los niveles de entropía en el sistema.

Palabras clave: camino, entropía, negentropía, usuario, vehículo.

\section{Abstract}

Context: The recent increase in accidents, mishaps or road accidents in urban systems with metropolitan characteristics has caused concern in the authorities responsible for planning the issue of mobility and transport subsystems. Indeed, there is convergence that the occurrence of this type of events is away from purely stochastic factors, but are the result of anomalies associated with the intrasystemic interaction of the vehicle, the user and the road. In this sense, the objective of this work is to determine through systemic analysis the components that are causing entropy in the second most important metropolis in Mexico.

Method: In this analysis the precepts of systems theory are used; in such a way that through the tringulation of data obtained in situ and solved documentarily the causal components of intrasystemic entropy are determined. To do this, a modified derivation of the Pressure-State-Response model that identifies homeostasis in the urban system is elaborated.

Results: The high exposure rates force the user to occupy the road subsystem for long periods of time, which triggers voluntary and involuntary human errors complemented by the lax requirements for obtaining driving licenses. From here, the components that produce accidents include in the road part the pavements in poor condition, absence of signaling, poor design of intersections, among others; in the vehicle part, mechanical failures due to the useful life of the internal components, flexible regulatory frameworks, and other; in the part of the user for breach of the regulations, physiological factors among others.

Conclusions: From the systemic analysis, mobility is a subject of valuable study to detect the imbalances in the trinomial Vehicle-User-Road (V-C-U), which lead us to generate preventive and corrective measures that suppress metropolitan accidents, morbidity and mortality in the metropolis; or in our terms to reduce entropy levels in the system.

Keywords: entropy, negentropy, road, user, vehicle.

\section{INTRODUCCIÓN}

Cotidianamente ocurren en el mundo cientos de miles de eventos relacionados con alguna anomalía en la interacción de los componentes intrasistémicos; a tal grado que, en el menor de los casos, la presencia de estos eventos genera costos económicos significativos en la infraestructura de transporte; sin embargo, también se tienen costos sociales de complejas magnitudes asociadas a la cuantificación y cualificación de lesionados y decesos. En este sentido, el accidente puede representar un "hecho no planeado ni controlado" (Mangosio, 2002). Sin embargo, según algunas posturas estos eventos no son aleatorios, sino que se deben a un conjunto de posibles combinaciones de los elementos asociados con el trinomio vehículo/ usuario/camino (VUC).

En función de lo anterior, la figura 1 muestra las cifras de la Organización Mundial de la Salud (2016) relacionada con el número de decesos por accidentes viales a nivel global.

Efectivamente, es posible observar en la figura anterior que aparentemente México, Brasil y algunos países adyacentes a este último, contenían índices importantes de mortalidad por accidentalidad vial en América Latina; sin embargo, al introducir el concepto de tasa de mortalidad por cada 100.000 habitantes, el panorama latinoamericano se vuelve alarmante en países como Ecuador, Bolivia o Paraguay; toda vez que conforme con la tabla 1 evidencian entropías en la interacción 


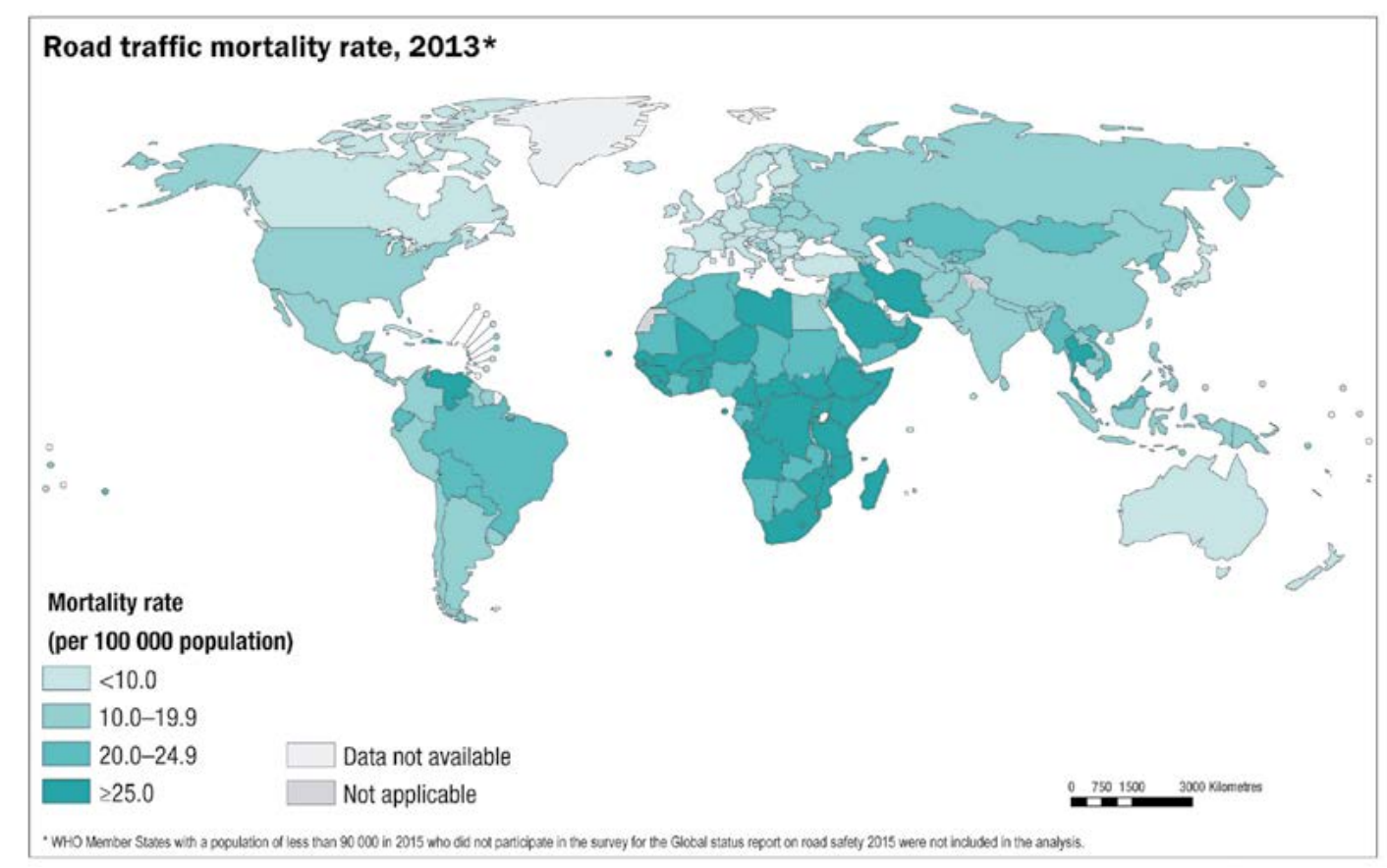

Figura 1. Número de decesos totales en accidentes de tránsito por país para el año 2013

Fuente: OMS (2016).

intrasistémica de los componentes constituyentes de la movilidad urbana.

De acuerdo con los datos de la OMS (2016), en Ecuador, Bolivia o Paraguay, la tasa de mortalidad por accidentes de tránsito contrastaba en 2013 con los indicadores de México, Chile y Argentina, los cuales muestran las menores tasas de mortalidad para 2013; no obstante, estos países aún se encuentran lejos de mostrar políticas públicas exitosas en la prevención de accidentes, como sí ocurre en Dinamarca, Noruega, Suecia, Inglaterra y otros, donde las tasas son iguales o menores a 6 decesos por cada 100.000 habitantes, como se muestra en la tabla 2.

Tabla 1. Índice de mortalidad en algunos países de América Latina para el año 2013

\begin{tabular}{|c|c|c|}
\hline País & Número de decesos & Tasa de mortalidad (\%) \\
\hline Argentina & 5619 & 13,6 \\
\hline Bolivia & 2476 & 23,2 \\
\hline Brasill & 46935 & 23,4 \\
\hline Chile & 2179 & 12,4 \\
\hline Colombia & 8107 & 16,8 \\
\hline Ecuador & 3164 & 20,1 \\
\hline México & 15062 & 12,3 \\
\hline Paraguay & 1408 & 20,7 \\
\hline Perú & 4234 & 13,9 \\
\hline Uruguay & 567 & 16,6 \\
\hline Venezuela & No hay datos & No hay datos \\
\hline
\end{tabular}

Fuente: elaboración propia con datos de la OMS (2016). 
Tabla 2. Índice de mortalidad en algunos países desarrollados para el año 2013

\begin{tabular}{|c|c|c|}
\hline País & Número de decesos & Tasa de mortalidad (\%) \\
\hline Alemania & 3540 & 4,3 \\
\hline Australia & 1252 & 5,4 \\
\hline Austria & 455 & 5,4 \\
\hline Canadá & 2114 & 6,0 \\
\hline Dinamarca & 196 & 3,5 \\
\hline Francia & 3268 & 5,1 \\
\hline Inglaterra & 1827 & 2,9 \\
\hline Italia & 3721 & 6,1 \\
\hline Japón & 5971 & 4,7 \\
\hline Noruega & 192 & 3,8 \\
\hline Suecia & 272 & 2,8 \\
\hline
\end{tabular}

Fuente: elaboración propia con datos de la OMS (2016).

En la mayoría de los países europeos se han implementado políticas públicas tendientes al desarrollo de hábitos de movilidad para la prevención de accidentes y la mitigación de morbilidad y mortalidad. En este sentido, en una prospección de la Comisión Europea (2014) desde 2010 hasta 2020, la tendencia de la mortalidad se torna decreciente (figura 2).

En el caso de México, el estado de Jalisco ha generado altas tasas de accidentalidad, morbilidad y mortalidad, las cuales están por encima de la media nacional mexicana respecto a estados que tienen mayor población y parque vehicular, como el Estado de México o la Ciudad de México. De acuerdo con el Secretariado Técnico del Consejo Nacional de Prevención de Accidentes (STCONAPRA), en 2015, la media de tasa de mortalidad fue de 14,6 y 13,4 defunciones por cada 100.000 habitantes en el país, mientras que en el estado de Jalisco se tuvieron tasas de mortalidad de 19,5 y 17,2. En este sentido, Ciudad de México presentó tasas inferiores, con el 8,7; casi la mitad de las presentadas por el estado de Jalisco (figura 3).

Figura 2. Prospección 2010-2020 de la mortalidad por accidentes viales en Europa

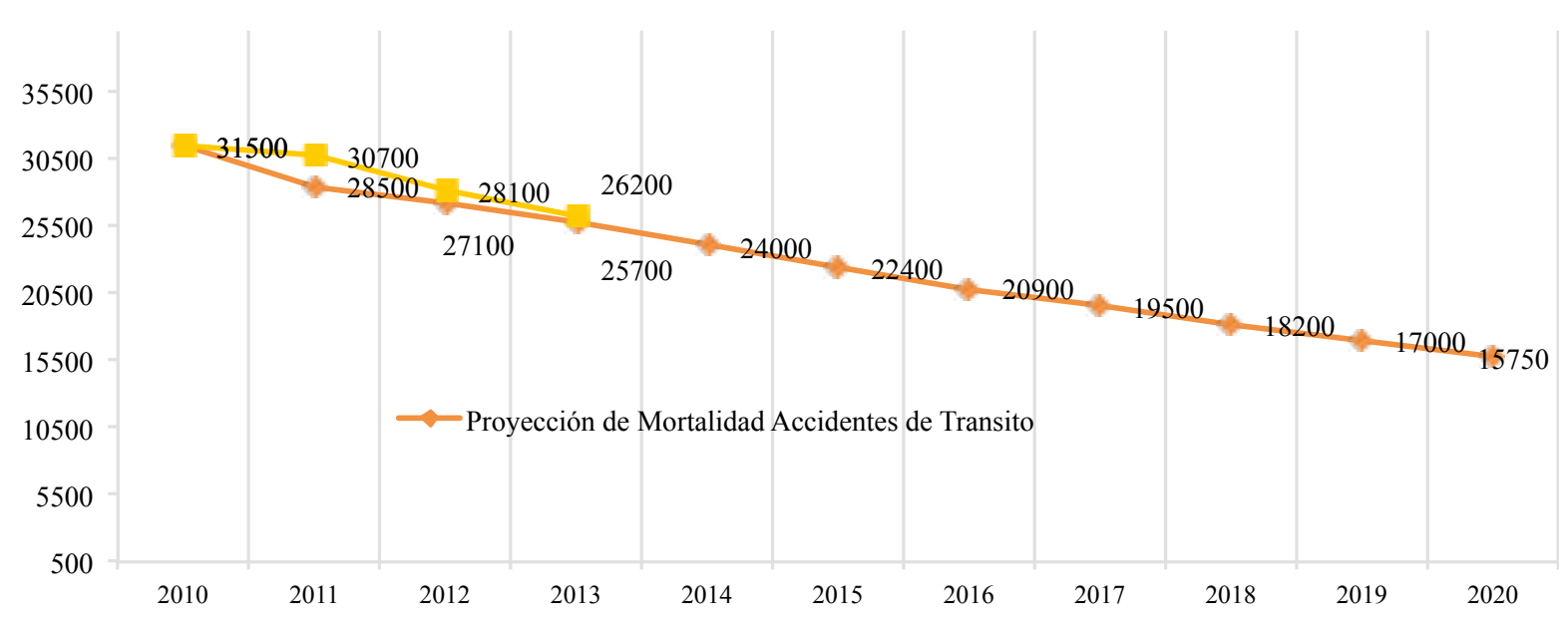

Fuente: Comisión Europea (2014) 
Por otro lado, el área metropolitana de Guadalajara perteneciente al estado de Jalisco, en los últimos años ha experimentado un descenso en las tasas de accidentalidad pasando de 15,40 fallecidos por cada 100.000 habitantes en 2012 a 10,88 en 2016, como se indica en la figura 4. Lo anterior se explica debido a la introducción de control en la velocidad a través de radares y del programa conocido como "Torito" que realiza una inspección de alcoholimetría en los principales ejes viales de la metrópoli.
Estudios realizados en Suecia, Inglaterra, Holanda, Estados Unidos, Australia y Nueva Zelanda, muestran que, a mayores tasas de motorización, existe un grado mayor de exposición a los accidentes (Marchesini y Weijermars, 2010; Johnston, 2010). Esto es evidente en Estados Unidos donde aún se mantienen altas tasas de mortalidad y morbilidad en accidentes de tránsito. Asimismo, Collazos (1982) encontró que Colombia, en una década, el parque vehicular había crecido casi dos

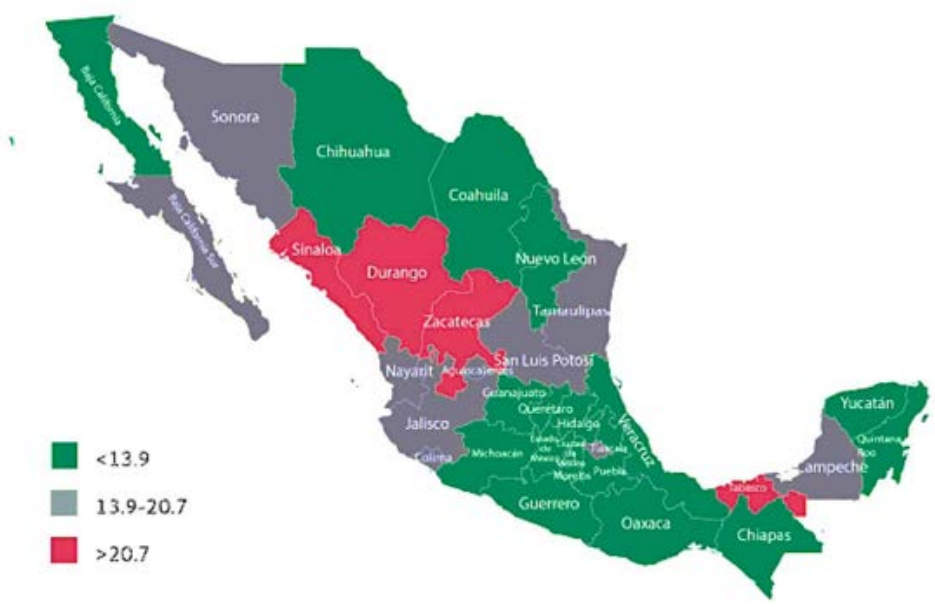

Figura 3. Tasa de mortalidad por entidad federativa en México en 2015

Fuente: STCONAPRA (2017).

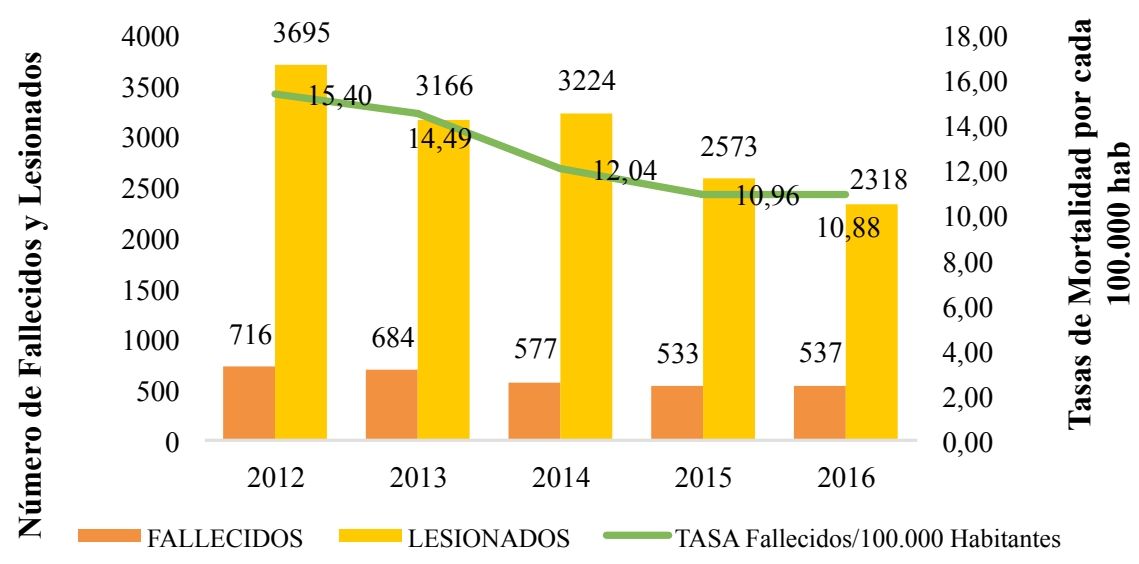

Figura 4. Fallecidos y lesionados por accidentes viales en el AMG 2012-2016

Fuente: elaboración propia, con datos de la SEMOV Jalisco 2017 
y media veces y el número de defunciones en accidentes de tránsito en más del $100 \%$.

En función de lo anterior, la Administración Federal de Carreteras (FHWA) realizó hace cerca de cuatro décadas un estudio exhaustivo de causalidad de accidentes de vehículos, y determinó que los errores humanos o deficiencias fueron la causa definitiva entre el $90 \%$ y el $93 \%$ de todos los casos analizados (Centro de Estudios para el Internet y la Sociedad de la Facultad de Derecho de la Universidad de Stanford, 2013). De igual forma, Hatakka et al. (2002) observaron que la participación de conductores inexpertos está asociada a un mayor número de accidentes de tránsito. Por su parte, Gregersen et al. (2000) también encontraron que retrasar permisos (licencias) para proveer mayores horas de práctica e incrementar las habilidades de manejo hasta convertirse en destreza, representa una disminución de accidentes en conductores inexpertos.

No obstante, varios años antes en Japón, Renge (1983) encontró una relación semejante a la encontrada en los países escandinavos; ya que, a mayores horas de práctica para la obtención de la licencia de manejo, se observó una disminución en accidentes y violaciones a las leyes de movilidad. Sin embargo, los conductores más hábiles que eran capaces de aprobar los requisitos de licenciamiento con pocas horas de práctica se ven comúnmente involucrados en accidentes e infracciones, lo que demuestra que el desarrollo del hábito de conducción segura es insuficiente con poca supervisión y práctica.

El Centro de Investigación de Accidentes de la Universidad Monash, en Australia (2005) identifica al error humano y a las capacidades disminuidas como el principal factor de accidentes con un valor del $95 \%$ en todos los choques y accidentes estudiados. Otro estudio publicado en Estados Unidos en 2001 reporta que los errores en el comportamiento humano fueron los causantes del $99 \%$ de todos los choques de vehículos investigados (Centro de Estudios para el Internet de la Facultad de Derecho de la Universidad de Stanford,
2013). En el caso de México, Cuevas, Pérez, Mayoral y Mendoza (2012) del Instituto Mexicano del Transporte reportaban que el error humano fue el causante de accidentes viales en un $73 \%$ a nivel nacional.

De Quevedo, Asprilla y González (2017) comentan que la carente planificación sistémica en la metrópoli de Guadalajara ha generado un escenario complejo con elementos que determinan en términos inciertos la morfología; sobre todo, porque la movilidad de los habitantes está supeditada al automóvil y a los autobuses públicos de bajo volumen, los cuales han contribuido con grandes externalidades (accidentalidad, mortalidad, morbilidad, congestionamiento vial, entre otras). En este sentido, el objetivo de este trabajo consiste en determinar por medio del análisis sistémico los componentes que están provocando entropía en la metrópoli de Guadalajara, México; para ello se hace énfasis en las determinantes del vehículo, usuario y camino. Esto con la intención de incidir en la adopción de mejores prácticas de prevención vial, tanto para tomadores de decisión, planificadores y población usuaria.

\section{METODOLOGÍA}

De acuerdo con Leplat (1982) y Cuny (1979), "un accidente es un síntoma de disfuncionamiento de un sistema" (p. 300), o en otros términos de ciertas anomalías en la interacción intrasistémica del trinomio vehículo/usuario/camino. En este sentido, en las interfaces entre los sistemas donde se producen la mayor cantidad de accidentes, ambos autores sugieren analizar los componentes del sistema y, particularmente, las zonas de intersección o las denominadas interfaces. Adicionalmente, las condiciones medioambientales pueden presentarse en forma de condiciones atmosféricas o climáticas que afecten la percepción o el intercambio de información en el sistema, como lluvia, niebla, nieve, calor excesivo, cambio de condiciones diurnas a nocturnas, ruido, luces brillantes y otros (Cal y Mayor y Cárdenas, 2010). 
Metodológicamente, el estudio se estructura en tres fases de análisis sistémico: a) análisis del vehículo, b) análisis del usuario y c) análisis del camino. Posteriormente, se aplica los fundamentos modificados del modelo presión/estado/respuesta (PER), identificando que la presión la ejercen los componentes entrópicos intrasistémicos en el trinomio vehículo/usuario/camino, que el estado es la homeostasis del sistema urbano o, en otros términos, las condiciones de accidentalidad y mortalidad, y la respuesta es el conjunto de acciones tendientes a reducir los niveles de entropía (componentes negentrópicos). Este modelo se fundamenta en las premisas del enfoque sistémico, con aplicabilidad en problemáticas de orden medioambiental; por tanto, provee un marco descriptivo a través de un conjunto de indicadores de intervención para mitigar los impactos antrópicos ejercidos en el ambiente. Esta metodología fue implementada por la Organización para la Cooperación y Desarrollo Económico (2002), con el objetivo de evaluar el estado del medio ambiente en sus países miembros (figura 5).

En este estudio se elabora un esquema considerando la estructura del modelo anterior: presión, estado y respuesta, pero trasladando la temática de la movilidad urbana y la accidentalidad en el sistema urbano de la metrópoli de Guadalajara, tal y como se muestra en la figura 6 .

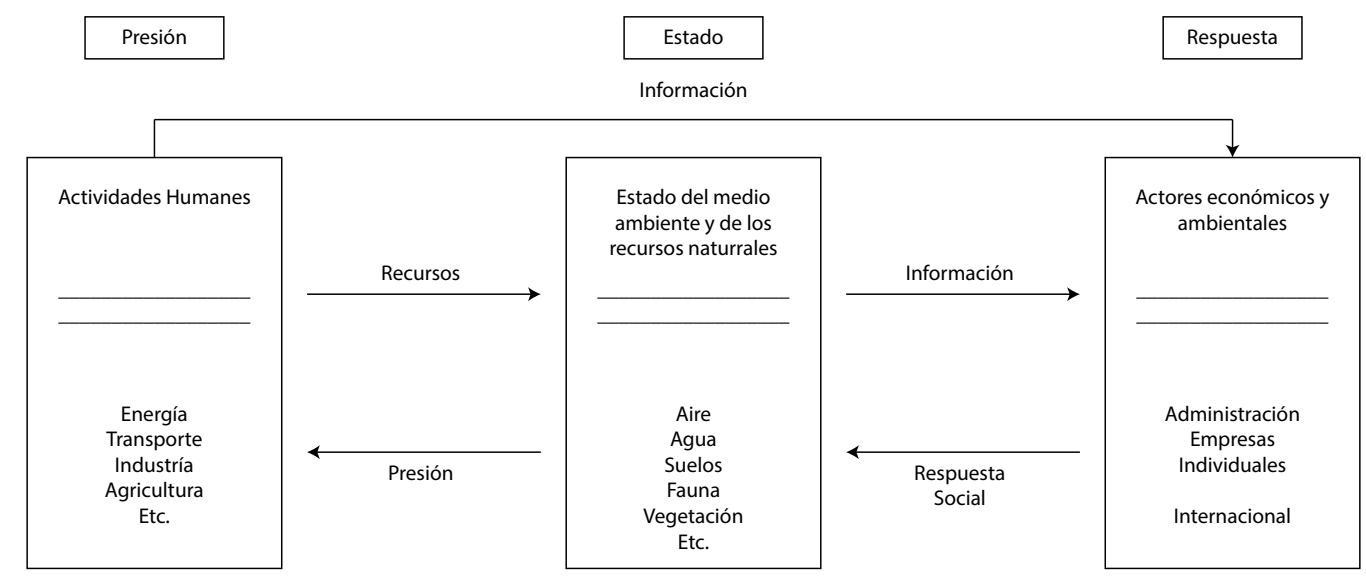

Respuestas Sociales (Decisiones - Acciones) Indicadores Ambientales

Figura 5. Estructura del modelo PER

Fuente: SIASEG (2012).

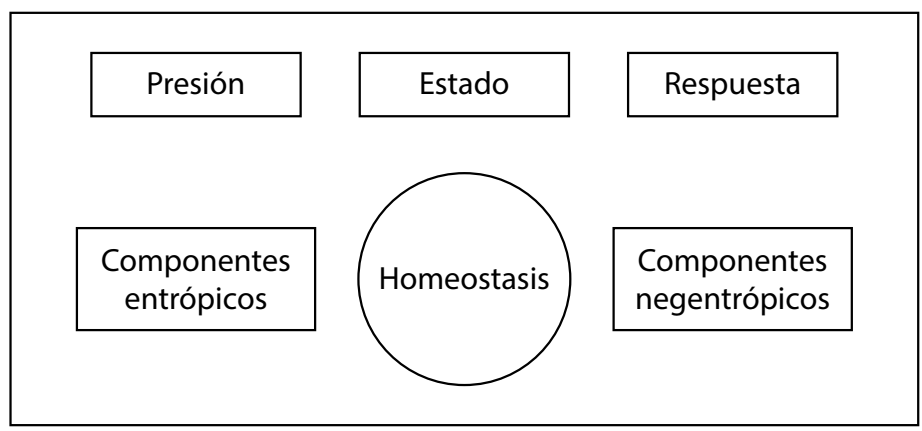

Figura 6. Esquema PER modificado

Fuente: elaboración propia. 


\section{RESULTADOS}

Desde el punto de vista sistémico, el congestionamiento vial crónico como lo denomina ONU-Hábitat (2015). En la metrópoli de Guadalajara genera altas tasas de exposición que obliga a conductores y ocupantes de diversos modos de transporte a ocuparlos por largos periodos de tiempo, lo cual detona en errores humanos voluntarios e involuntarios asociados con el incremento de la velocidad, el sobre calentamiento del vehículo o el sistema de frenado necesario para proveer seguridad (García, Acosta y Flórez, 2015, p. 53). Sin embargo, el estado de Jalisco como la mayoría de los estados de México, tiene requisitos muy laxos para la obtención de una licencia de manejo de un automóvil.

Los requisitos se resumen en presentar documentación relacionada con la clave única de registro de población (CURP), identificación oficial, comprobante de domicilio y tipo de sangre. Adicionalmente, se requiere aprobar examen de conocimientos viales y examen práctico de manejo, y realizar el pago correspondiente (Gobierno del Estado de Jalisco, 2017). Sin embargo, no existen programas formativos formales de educación vial y el desarrollo de estas habilidades se da bajo la supervisión de padres, hermanos mayores, y para una minoría de la población por medio de instructores especializados que brindan sus servicios por medio de empresas privadas.

En la actualidad, los planes educativos no incluyen en ningún nivel cursos enfocados al desarrollo de habilidades para la movilidad; de tal forma que no sorprende que muchos conductores habituales no hayan alcanzado ni siquiera el nivel de habilidades de expertos presentado por Falkmer y Gregersen (2001). De aquí, se desprende que el desconocimiento de las leyes de movilidad y la falta de experiencia y como consecuencia de habilidades mínimas para la conducción estén ausentes y den como resultados causales que producen errores y accidentes, tal y como se observa en las figuras 7 y 8 .

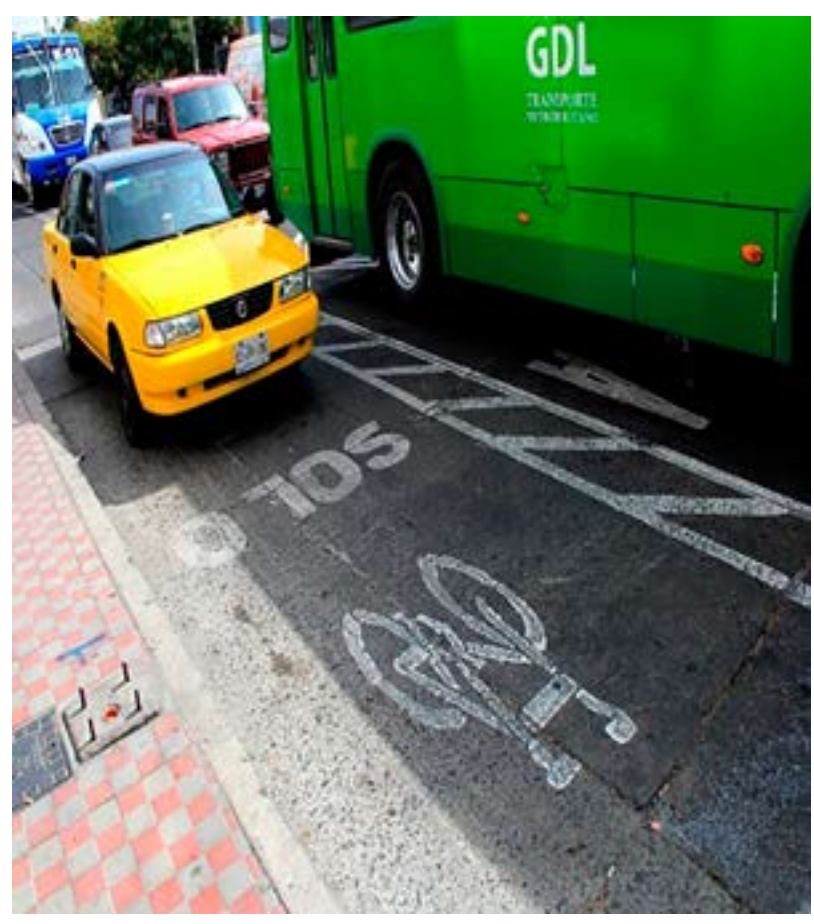

Figura 7. Invasión de ciclovías

Fuente: Periódico Máspormás (2016)

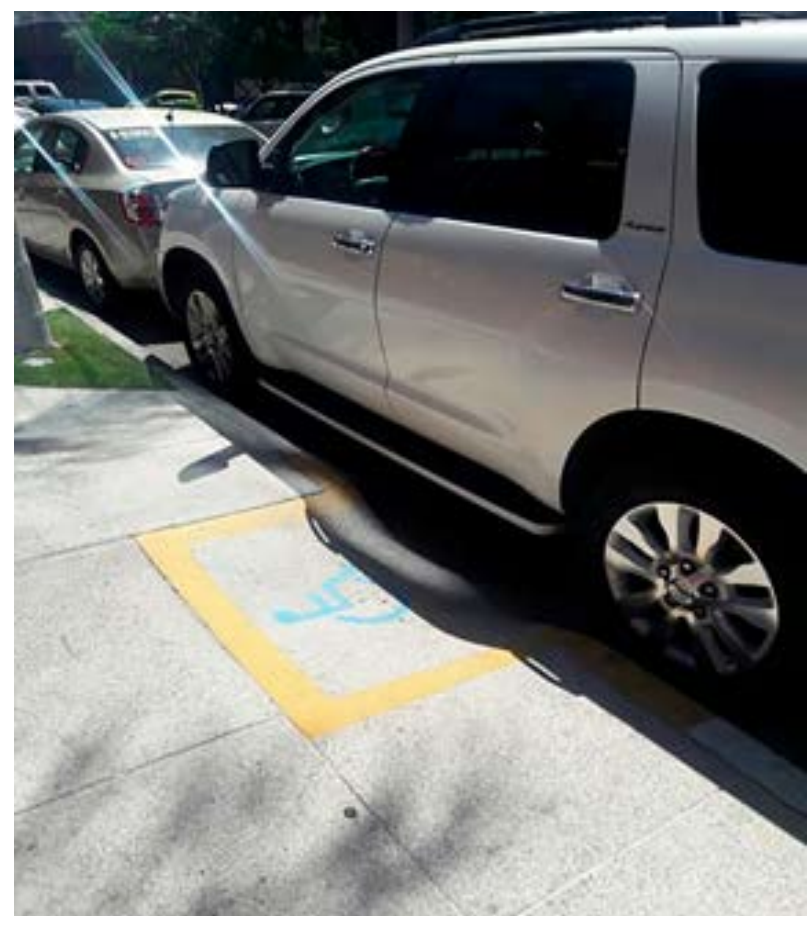

Figura 8. Obstrucción de acceso

Fuente: Nacho García (vía Twitter, 2017). 
El error humano también está influenciado por el medio ambiente y el mantenimiento deficiente de la infraestructura como elemento básico. No obstante, en la metrópoli una grave problemática son los pavimentos en mal estado, que obligan a los conductores a reaccionar de manera imprevista para evitar daños a sus vehículos, por lo general, estas maniobras evasivas implican movimientos súbitos con invasión frecuente de otros carriles o frenadas de emergencia que ocasionan colisiones traseras. En la figura 9 se muestra un pavimento que exhibe grandes baches y deformación superficial.

En este orden de ideas, la metrópoli de Guadalajara aglutina en su extensa red vial casi dos millones de vehículos de pasajeros y de carga; y más de cinco mil autobuses urbanos. Según el Diario Oficial de la Federación (2012), el parque vehicular en México promedia los 16,3 años, lo cual representa una flota de vehículos envejecida en la cual la posibilidad de fallas en el elemento máquina sea algo muy factible; sobre todo, porque la mayoría de estos vehículos emplean sistemas de frenado de baja y media eficiencia (sistemas disco/tambor) aunado a sistemas de suspensión y dirección con alto deterioro; inclusive, el equipamiento de sistemas de frenado antibloqueo (ABS), de asistencia al frenado (BAS), control de tracción y bolsas de aire todavía no es obligatorio ni siquiera en automóviles nuevos, conforme se puede observar en la figura 10.

Los efectos del uso de distractores en la movilidad constituyen en sí un cuerpo de conocimiento a ser desarrollado en extenso; sin embargo, algunas ciudades en México empiezan a arrojar ciertas cifras de esta perniciosa relación, por ejemplo, la ciudad de Culiacán en el estado de Sinaloa. El coordinador del área de prevención de accidentes de Cruz Roja en ese municipio estima que $54 \%$ de los accidentes de tránsito se han dado por el uso de teléfonos inteligentes como distractores durante la conducción (López, 2017). Igualmente, a estas conductas riesgosas, la intención de movilizarse en estado de ebriedad o intoxicación por alguna sustancia legal o ilegal es algo frecuente, no solo en conductores de automóviles, autobuses y choferes del transporte de carga sino en ciclistas e inclusive peatones (tablas 3 y 4 ).

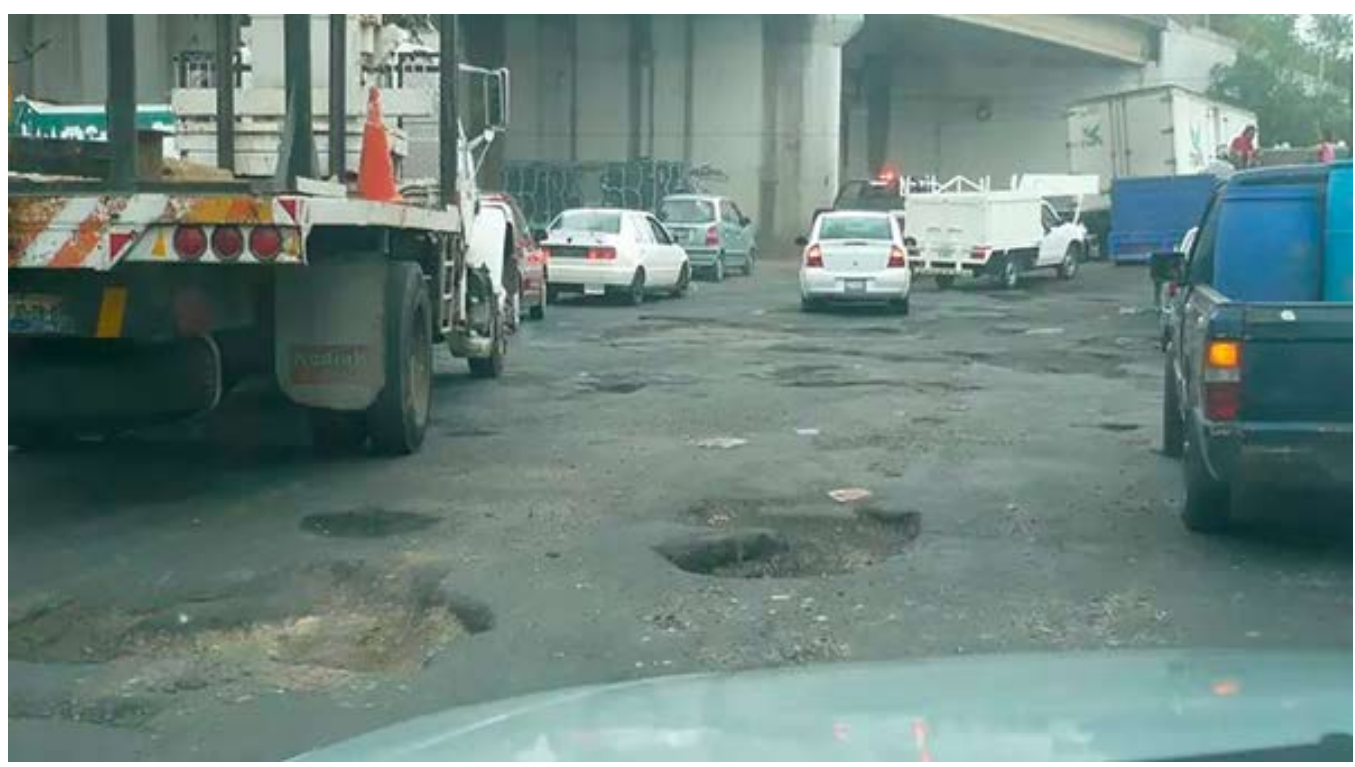

Figura 9. Grave deterioro de pavimento en la metrópoli de Guadalajara

Fuente: elaboración propia. 


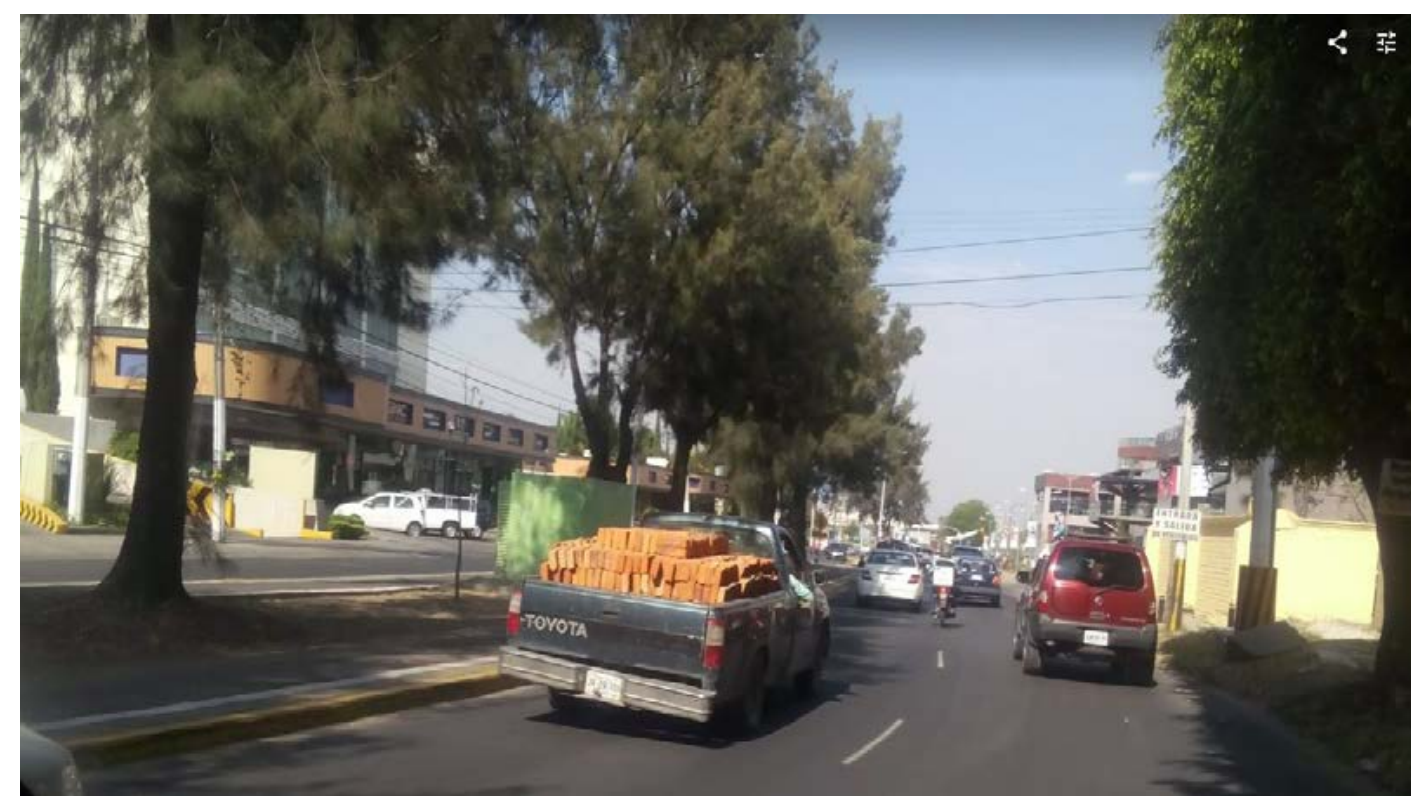

Figura 10. Vehículo con más de 15 años de antigüedad

Fuente: elaboración propia.

Tabla 3. Defunciones en choques de conductores entre 18 y 34 años en la metrópoli de Guadalajara del 1-01-2012 al 31-03-2017

\begin{tabular}{lllllll}
\hline \multicolumn{1}{c}{ Municipio } & $\begin{array}{c}\text { Total de } \\
\text { Autopsias }\end{array}$ & $\begin{array}{c}\text { Positivos } \\
\text { de } \\
\text { alcohol }\end{array}$ & $\begin{array}{c}\text { \% de autopsias } \\
\text { positivas de } \\
\text { alcohol }\end{array}$ & $\begin{array}{c}\text { Positivos de } \\
\text { toxicologico }\end{array}$ & $\begin{array}{c}\text { \% de autopsias } \\
\text { positivas otras } \\
\text { substancias }\end{array}$ & $\begin{array}{c}\text { \% de autopsias } \\
\text { positivas a alcohol } \\
\text { u otras substancias }\end{array}$ \\
\hline El Salto & 20 & 6 & $30,0 \%$ & 2 & $10,0 \%$ & $40,0 \%$ \\
\hline Guadalajara & 306 & 47 & $15,4 \%$ & 27 & $8,8 \%$ & $24,2 \%$ \\
\hline $\begin{array}{l}\text { Ixtlahuacán de los } \\
\text { Membrillos }\end{array}$ & 5 & 1 & $20,0 \%$ & 0 & $0,0 \%$ & $20,0 \%$ \\
\hline $\begin{array}{l}\text { Tlajomulco de } \\
\text { Zúñiga }\end{array}$ & 65 & 16 & $24,6 \%$ & 1 & $1,5 \%$ & $26,2 \%$ \\
\hline Tlaquepaque & 35 & 7 & $20,0 \%$ & 0 & $0,0 \%$ & $20,0 \%$ \\
\hline Tonalá & 25 & 11 & $44,0 \%$ & 2 & $8,0 \%$ & $52,0 \%$ \\
\hline Zapopan & 89 & 34 & $38,2 \%$ & 2 & $2,2 \%$ & $40,4 \%$ \\
\hline Zapotlanejo & 26 & 11 & $42,3 \%$ & 0 & $0,0 \%$ & $42,3 \%$ \\
\hline Juanacatlan & 0 & 0 & $0,0 \%$ & 0 & $0,0 \%$ & $0,0 \%$ \\
\hline Totales & 571 & 133 & $23,3 \%$ & 34 & $6,0 \%$ & $29,2 \%$ \\
\hline
\end{tabular}

Fuente: elaboración propia, a partir de Instituto Jalisciense de Ciencias Forenses (2017).

El Gobierno de Jalisco ha emprendido una cruzada para sancionar a conductores ebrios desde noviembre de 2013 a la fecha. El último reporte de la Secretaría de Movilidad (SEMOV), concentra información desde noviembre del 2013 a enero de 2017. Estos datos, se presentan en la figura 11, con un promedio de 26,53 pruebas positivas, $y$ con una tendencia a la baja, sin embargo, en muchos meses se exceden estos valores. 
Tabla 4. Defunciones por volcamientos de conductores entre los 18 y 34 años en la metrópli de Guadalajara del (1-01-2012 al 31-03 2017)

\begin{tabular}{lcccccc}
\hline \multicolumn{1}{c}{ Municipio } & $\begin{array}{c}\text { Total de } \\
\text { Autopsias }\end{array}$ & $\begin{array}{c}\text { Positivos } \\
\text { de } \\
\text { alcohol }\end{array}$ & $\begin{array}{c}\text { \% de autopsias } \\
\text { positivas de } \\
\text { alcohol }\end{array}$ & $\begin{array}{c}\text { Positivos de } \\
\text { toxicologico }\end{array}$ & $\begin{array}{c}\text { \% de autopsias } \\
\text { positivas otras } \\
\text { substancias }\end{array}$ & $\begin{array}{c}\text { \% de autopsias } \\
\text { positivas a alcohol } \\
\text { u otras substancias }\end{array}$ \\
\hline El Salto & 11 & 5 & $45,5 \%$ & 0 & $0,0 \%$ & $45,5 \%$ \\
\hline Guadalajara & 84 & 22 & $26,2 \%$ & 9 & $10,7 \%$ & $36,9 \%$ \\
\hline $\begin{array}{l}\text { Ixtlahuacán de } \\
\text { los Membrillos }\end{array}$ & 8 & 3 & $37,5 \%$ & 1 & $12,5 \%$ & $50,0 \%$ \\
\hline $\begin{array}{l}\text { Tlajomulco de } \\
\text { Zúñiga }\end{array}$ & 27 & 17 & $63,0 \%$ & 4 & $14,8 \%$ & $77,8 \%$ \\
\hline Tlaquepaque & 12 & 7 & $58,3 \%$ & 0 & $0,0 \%$ & $58,3 \%$ \\
\hline Tonalá & 7 & 2 & $28,6 \%$ & 0 & $0,0 \%$ & $28,6 \%$ \\
\hline Zapopan & 35 & 11 & $31,4 \%$ & 0 & $0,0 \%$ & $31,4 \%$ \\
\hline Zapotlanejo & 23 & 14 & $60,9 \%$ & 0 & $0,0 \%$ & $60,9 \%$ \\
\hline Juanacatlan & 0 & 0 & $0,0 \%$ & 0 & $0,0 \%$ & $0,0 \%$ \\
\hline Totales & 207 & 81 & $39,1 \%$ & 14 & $6,8 \%$ & $45,9 \%$ \\
\hline
\end{tabular}

Fuente: elaboración propia a partir de Instituto Jalisciense de Ciencias Forenses (2017).

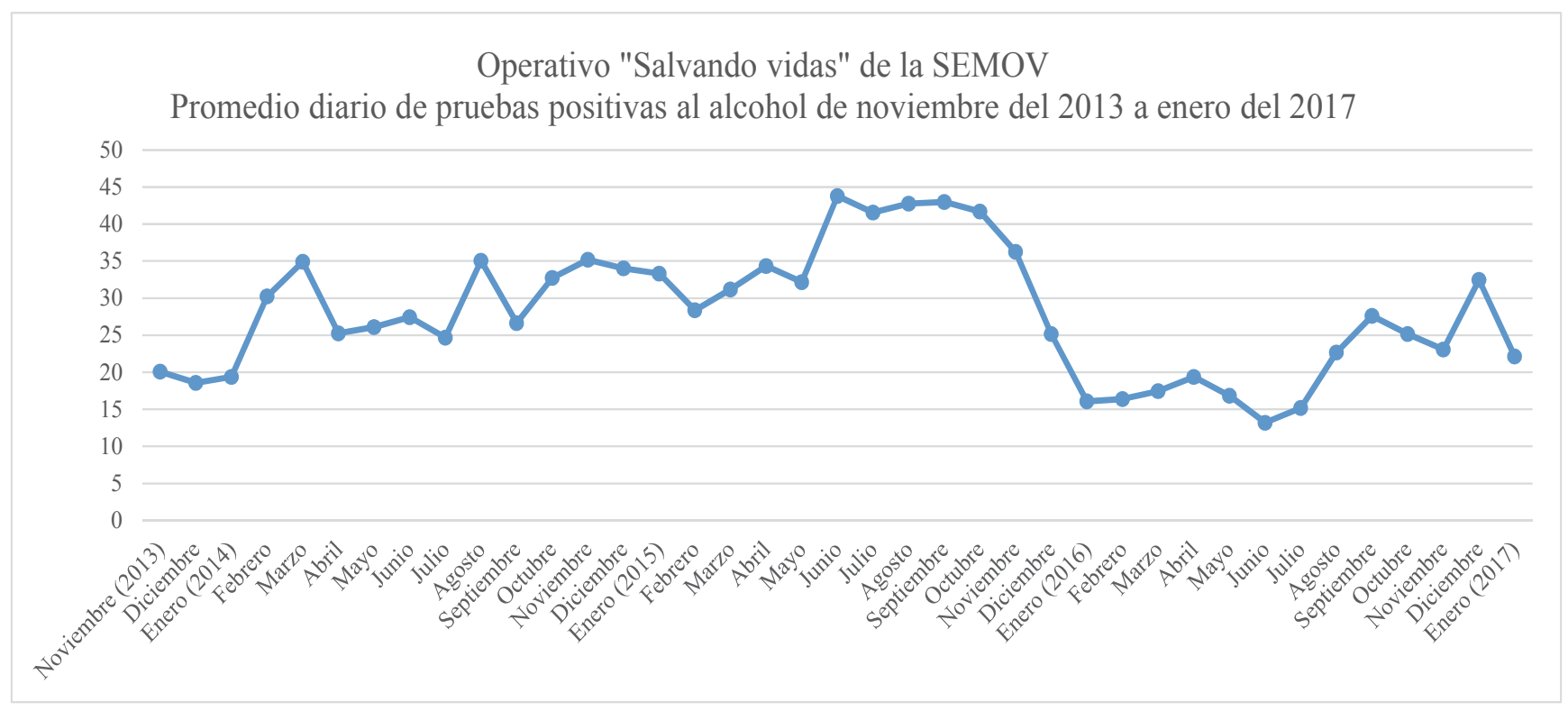

Figura 11. Promedio diario de pruebas positivas de alcoholemia por mes 2013 a 2017

Fuente: elaboración propia con datos de la SEMOV (2017). 
El operativo Salvando Vidas de la SEMOV solo tiene la capacidad de detección de alcohol en el aliento y en la sangre; por tanto, conductores con consumo de sustancias tóxicas no son detectados por este programa.

En la metrópoli, en su sistema de movilidad presenta anomalías en todo el trinomio (VUC), y no es de sorprender que las altas tasas de accidentalidad, morbilidad y mortalidad; pues, esta última es la primera causa de muerte en jóvenes entre los 15 y 24 años; y la segunda entre los 5 y 14 años y también entre los 25 y 34 años (INEGI, 2015).

En la figura 12 se indican los componentes entrópicos y negentrópicos que se han determinado en función del trinomio VUC en la metrópoli de Guadalajara.

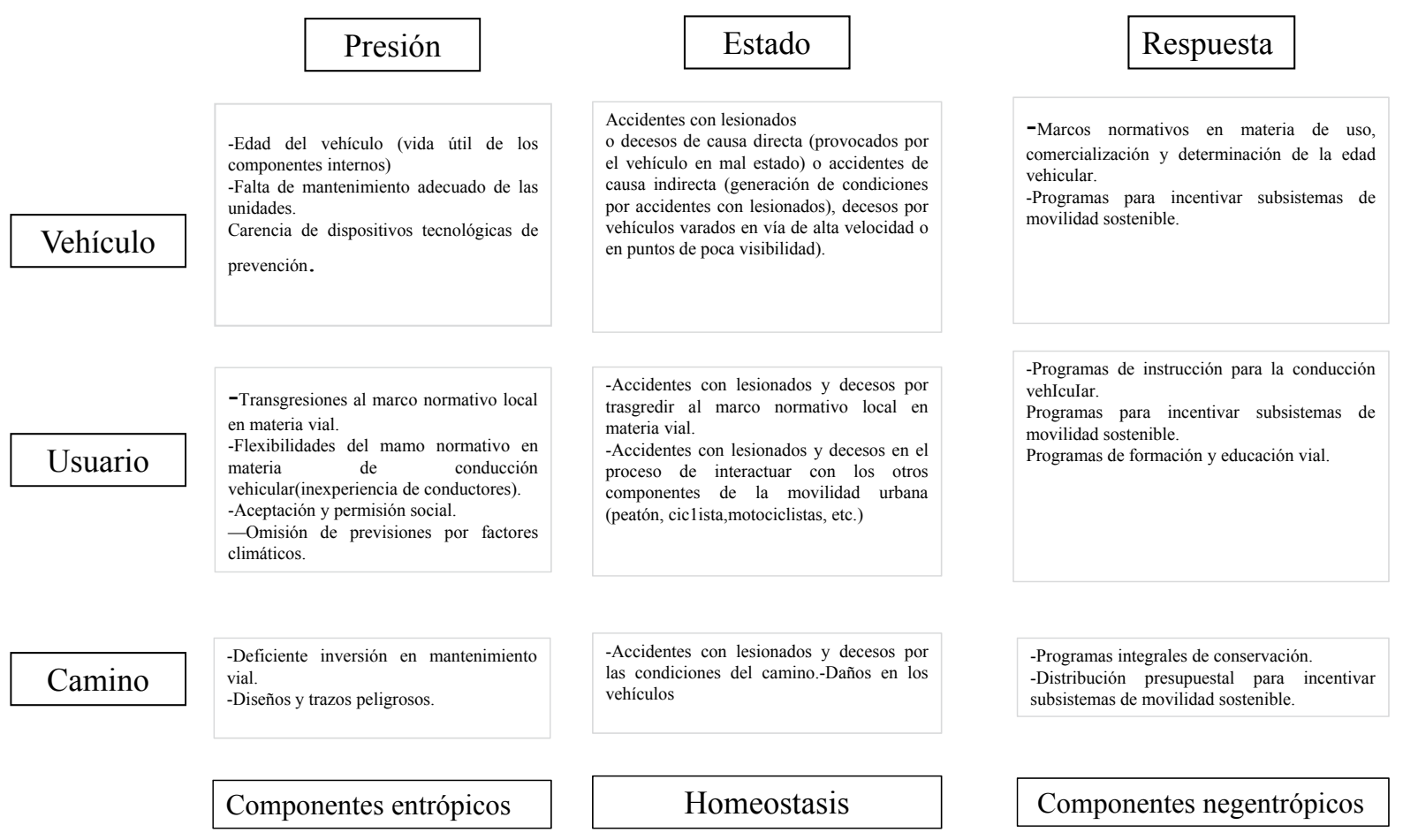

Figura 12. El modelo PER aplicado al trinomio VUC

Fuente: elaboración propia.

\section{CONCLUSIONES}

Es evidente que México, al igual que diversos países de Latinoamérica deben redoblar sus esfuerzos en mecanismos de respuesta en materia de movilidad urbana con un especial énfasis en la incentivación y consolidación de programas de movilidad sostenible; sin embargo, en el ejercicio de la movilidad motorizada es prioritario establecer marcos que instruyan de manera profunda a los futuros conductores en el proceso de la circulación de vehículos; así como también la supervisión continua del tránsito de vehículos viejos.

En la metrópoli de Guadalajara, la convivencia cotidiana entre los cerca de 5 millones de habitantes y los poco más de dos millones de vehículos ha generado fricciones intrasistémicas, sobre todo cuando la presencia vehicular se impone y se prioriza versus los otros sujetos de la movilidad. Esto implica que, en las relaciones entre los usuarios, 
los modos de transporte y el medio ambiente urbano se origina entropía, manifiesta en una homeostasia de incrementos de accidentalidad y con ellos lesionados y decesos.

No obstante, la tarea no es fácil, ya que las cifras presupuestales destinadas a la construcción de vialidades, obras complementarias para la agilización vehicular favorecen las altas tasas de motorización, y estas a la vez detonan en una mayor demanda que satura más pronto que tarde la capacidad de carga de las vías urbanas. Además, la utilización de vehículos envejecidos, carentes de mantenimiento preventivo y ausentes de dispositivos de seguridad tecnológica complican aún más la homeostasia del sistema urbano.

En términos sistémicos, el subsistema vial experimenta entropía no solo por la operatividad del propio sistema a través del desgaste vehicular inducido, sino por la presencia de fuerzas externas como el clima que van deteriorando su funcionamiento, pero que también se suman al error humano con infraestructura complementaria insuficiente para el desalojo del agua pluvial, o procesos constructivos y materiales de mala calidad. Adicionalmente, se presentan conductas sociales que suprimen la señalética o el equipamiento urbano; lo cual reduce el margen visual preventivo. Sobre esto último, hay que precisar que la ausencia de conocimiento, falta de capacitación y desarrollo de habilidades en la conducción motorizada fungen como componentes precursores de accidentes; en otros términos, como componentes entrópicos que omiten las restricciones normativas como los límites de velocidad, el uso inadecuado o no uso de equipo de seguridad y la intoxicación por consumo de bebidas alcohólicas o sustancias durante el ejercicio de la movilidad motorizada.

Recientemente, se han implementado estrategias negentrópicas a nivel municipal y estatatal, a través de diversos programas enfocados en la prevención de accidentes como lo son: banquetas libres y cruceros seguros, o el programa Salvando Vidas con diversos ejes como el control de alcoholimetría, sancionar los excesos de velocidad, uso del casco uso del cinturón de seguridad. Adicionalmente, todos los niveles de gobierno incluyendo al federal han realizado esfuerzos para mejorar la infraestructura para la movilidad. Sin embargo, sigue faltando un tema crucial que es la educación vial y sobre todo la consolidación de un tema todavía germinal en diversas urbes de México: la movilidad sustentable. La educación para la movilidad urbana sustentable, debe ser coordinada por la autoridad, pero solo será fructífera si las instituciones educativas desde los niveles básicos hasta la universidad se comprometan con educar y desarrollar habilidades y valores en los usuarios de la movilidad que centren sus principios en la inclusión social, la sustentabilidad y el respeto a todos los componentes intrasistémicos. Finalmente, desde el análisis sistémico la movilidad es una temática de estudio valiosa para detectar los desequilibrios en el trinomio VUC, que nos Ileven a generar medidas preventivas y correctivas que supriman la accidentalidad, la morbilidad y la mortalidad en la metrópolis de Guadalajara; o en nuestros términos para reducir los niveles de entropía en el sistema.

El estado actual del sistema de movilidad urbana en la metrópoli de Guadalajara (homeostasis) se encuentra en desequilibrio dinámico, debido a que la entropía (desestabilización sistémica) generada por la industria inmobiliaria y su cadena de distribución, la industria automotriz y de autopartes, los proveedores del servicio de transporte, las empresas de venta de combustibles y lubricantes, y en general los intereses de los grupos que ostentan temporalmente la representación social son mayores a la capacidad de carga del sistema, y por tanto, rebasan al conjunto de fuerzas tendientes a generar neguentropía (estabilización sistémica).

\section{REFERENCIAS}

Cal y Mayor, R. y Cárdenas, J. (2010). Ingeniería de transito: fundamentos y aplicaciones. Alfa Omega: México.

Centro de Investigación de Accidentes de la Universidad Monash (2005). Human error and road 
transport. Australia. Recuperado de http://www. monash.edu/_data/assets/pdf_file/0010/216946/ muarc256.pdf

Centro de Estudios para el Internet y la Sociedad de la Facultad de Derecho de la Universidad de Stanford (2013). Human error as a cause of vehicle crashes. EE. UU. Recuperado de http://cyberlaw.stanford.edu/ blog/2013/12/human-error-cause-vehicle-crashes

Collazos P., H. (1982). Epidemiología de los accidentes de tránsito en las principales ciudades de Colombia. Ingeniería e Investigación, 40-45. Colombia. Recuperado de https://dialnet.unirioja.es/descarga/ articulo/4902517.pdf

Comisión Europea (2014). Working together for safer roads in Europe: 10 highlights of Commission road safety work 2010-2014. Bélgica. Recuperado de http://europa.eu/rapid/press-release_MEMO-14-245_en.htm

Cuevas, A.C., Pérez, J.G., Mayoral, E.F. y Mendoza, A. (2015). Anuario Estadístico de Accidentes en Carreteras Federales (2013). México: Instituto Mexicano del Transporte. Recuperado de http://www.imt.mx/archivos/Publicaciones/DocumentoTecnico/dt61.pdf

Cuny, X. (1979). Les Accident de Travail. PUF París: Recuperado de https://www.sciencedirect.com/ science/article/pii/0376634982900402

De Quevedo, F., Asprilla, Y. y González, M. (2017). Entropías de la movilidad urbana en el espacio metropolitano de Guadalajara: transporte privado y calidad del aire. Revista Tecnura, 21(53), 138-149. Recuperado de https://revistas.udistrital.edu.co/ojs/ index.php/Tecnura /article/view/10725/13025

Diario Oficial de la Federación (2012). Programa Nacional para el Aprovechamiento Sustentable de la Energía 2009-2012. México. Recuperado de http://dof. gob.mx/nota_detalle_popup.php?codigo=5121860

Falkmer, T. y Gregersen, N. (2001). The Trainer ProjectDevelopmnent of a New Cost-Effective Pan-European Driver Training Methodology and How to Evaluate it. Proceeeding of the Conference: Road Safety in Three Continents. Rusia.

García, R.A., Acosta, M.A. y Flórez, E. (2015). Análisis del comportamiento de los frenos de disco de los vehículos a partir de la aceleración del proceso de corrosión. Tecnura, 19(45), 53-63.
Gobierno del Estado de Jalisco (2017). Trámites y servicios en línea. Licencia Nueva de automovilista. México. Recuperado de http://tramites.jalisco.gob.mx/ tramite/8358

Gregersen, N.P., Berg, H.Y., Engström, I., Nolén, S., Nyberg, A. y Rimmö, P.A. (2000). Sixteen years age limit for learner drivers in Sweden: an evaluation of safety effects. Accident Analysis and Prevention, 32(1), 25-35. Recuperado de https://doi. org/10.1016/S0001-4575(99)00045-7

Hatakka, M., Keskinen, E., Gregersen, N.P., Glad A. y Hernetkosk, K. (2002). From control of the vehicle to personal self-control; broadening the perspectives to driver education. Transportation Research Part F, 5(3), 201-215. Recuperado de https://doi. org/10.1016/S1369-8478(02)00018-9

Instituto Jalisciense de Ciencias Forenses (2017). Sistema estadístico sobre autopsias. Recuperado de http://cienciasforenses.jalisco.gob.mx/estadisticas_ sist1.php

Instituto Nacional de Estadística, Geografía e Informática (INEGI) (2015). Transporte. Índice de motorización por entidad federativa, 2000-2014. Recuperado de http://www3.inegi.org.mx/sistemas/ sisept/default.aspx? $\mathrm{t}=$ mamb137 \&s=est \&c=21690

Johnston, I. (2010). Beyond "best practice" road safety thinking and systems management. Safety Science, 9(48), 1175-1181. Recuperado de https://doi.org/10.1016/j.ssci.2009.12.003

Lepalt, J. (1982). Accidents and incidents production: Methods of analysis. Journal of Occupational Accidents, 4(2-4), Pages 299-310. https://doi. org/10.1016/0376-6349(82)90040-2

López, T. (2017). El uso de teléfono inteligente es la principal causa de accidentes en Culiacán. AND Informativo. Recuperado de http://adnportal.mx/ el-uso-de-telefono-inteligente-es-la-principal-causa-de-accidentes-en-culiacan/

Mangosio, J. (2002). Investigación sobre Accidentes. Buenos Aires: Universidad Católica de Argentina, Facultad de Ciencias Fisicomatemáticas e Ingeniería.

Marchesini, P. y Weijermars, W. (2010). The relationship between road safety and congestion on motorways. 
Países Bajos: Institute for Road Safety Research. Recuperado de https://www.swov.nl/sites/default/files/ publicaties/rapport/r-2010-12.pdf

Máspormás (2016). Deja la ciclovía, o paga. Recuperado de https://www. maspormas.com/2016/05/12/ deja-la-ciclovia-paga/

Organización para la Cooperación y Desarrollo Económico (OCDE) (2002). Rumo a um Desenvolvimento Sustentável Indicadores Ambientais: indicadores Ambientais. Série Cadernos de Referência. Salvador, Brasil.

Organización Mundial de la Salud (OMS) (2016). Road tráffic mortality rate, 2013. Recuperado de http:// gamapserver.who.int/mapLibrary/Files/Maps/Global_RoadTraffic_Mortality_2013.png

Organización de las Naciones Unidas (ONU-Hábitat) (2015). Reporte nacional de movilidad urbana en México 2014-2015. Recuperado de http://conurbamx.com/home/wp-content/uploads/2015/07/
Reporte-Nacional-de-Movilidad-Urbana-en-Mexico-2014-2015-Final.pdf

Renge, K. (1983). Junge Kraftfahrer in Japan. Bereich Unfallforschung, Alemania: Bundesanstalt für Straßenwesen.

Secretariado Técnico del Consejo Nacional para la Prevención de Accidentes (STCONAPRA) (2017). Perfil nacional y perfiles estatales de seguridad vial. Recuperado de https://www.gob.mx/cms/uploads/ attachment/file/242263/Jalisco.pdf

Secretaria de Movilidad del Estado de Jalisco (SEMOV) (2017). Reporte Salvando Vidas. Recuperado de http://semov.jalisco.gob.mx/sites/semov.jalisco. gob.mx/files/osv_acumulado_2013-2017.pdf

Sistema de Indicadores Ambientales y de Sustentabilidad (Siaseg) (2012). Modelo P-E-R. México: Instituto de Ecología, Gobierno de Guanajuato. Recuperado de http://ecologia.guanajuato.gob.mx/sitio/micro/ siaseg/modeloper.php

\section{(c) $(1) \Theta \Theta$}

